\title{
Radiographic anatomy and clinical significance of percutaneous endoscopic transforaminal oblique fixation from posterior corner in lumbar spine
}

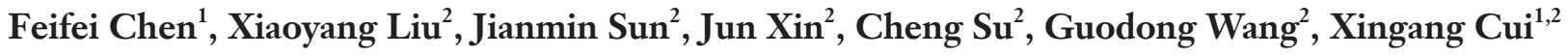 \\ ${ }^{1}$ Department of Spine Surgery, Shandong Provincial Hospital, Cheeloo College of Medicine, Shandong University, Jinan, China; ${ }^{2}$ Department of \\ Spine Surgery, Shandong Provincial Hospital Affiliated to Shandong First Medical University, Jinan, China \\ Contributions: (I) Conception and design: X Cui, F Chen; (II) Administrative support: None; (III) Provision of study materials or patients: X Liu, G \\ Wang; (IV) Collection and assembly of data: J Xin, C Su; (V) Data analysis and interpretation: F Chen, X Liu, J Sun; (VI) Manuscript writing: All \\ authors; (VII) Final approval of manuscript: All authors. \\ Correspondence to: Xingang Cui, MD. Department of Spine Surgery, Shandong Provincial Hospital Affiliated to Shandong University, Shandong \\ Provincial Hospital Affiliated to Shandong First Medical University, No. 9677, Jingshi Road, Jinan, China. Email: spine2014@163.com.
}

\begin{abstract}
Background: Endoscopic transforaminal decompression and interbody fusion (ETDIF) has been widely discussed due to its advantages of less trauma, less bleeding, quick recovery, high safety, and relatively fewer complications, as well as adverse factors such as incomplete decompression, steep learning curve, low fusion rate, and high radiation risk. Furthermore, this technique requires the use of supplemental posterior pediclescrew. Decompression, interbody fusion and percutaneous pedicle screw implantation are not completed in a single channel. Percutaneous endoscopic transforaminal oblique fixation from posterior corner in lumbar spine (PETOFPC) overcomes the above limitations. The purpose of this study is to confirm the anatomical feasibility for PETOFPC in the posterolateral transforaminal approach and to provide anatomic data for the design of new integrated fixable and fused interbody cage.

Methods: Sixty volunteers (22 men and 38 women) who underwent lumbar CT scans were collected and sent to the GEAW4.4 workstation. As a cohort study, the distances and angles of each path in the sagittal and axial planes were measured and analyzed statistically.

Results: The lengths of each path are not less than $40 \mathrm{~mm}$, and the longest can be up to $46 \mathrm{~mm}$. The paths in full-length group are about $5 \mathrm{~mm}$ longer than that in medium group. $\mathrm{PE}$ (from point $\mathrm{P}$ to target $\mathrm{E}$ ) path was the optimal path. The angles of each path were significantly different $(\mathrm{P} \leq 0.001)$, namely, a1 $>\mathrm{a} 2>\mathrm{a} 3, \mathrm{~b} 1$ $>\mathrm{b} 2>\mathrm{b} 3$, and $\mathrm{c} 1<\mathrm{c} 2<\mathrm{c} 3$.

Conclusions: This study confirms anatomic feasibility for PETOFPC and provides anatomic data for the design of new integrated fixable and fused interbody cage. PETOFPC may be a very promising technology and have great clinical significance.
\end{abstract}

Keywords: Radiographic anatomy; lumbar spine; oblique fixation; posterior corner

Submitted Feb 28, 2020. Accepted for publication Sep 04, 2020.

doi: 10.21037/atm-20-2046

View this article at: http://dx.doi.org/10.21037/atm-20-2046

\section{Introduction}

Open lumbar interbody fusion is an effective method to restore the stability of the spine and correct the abnormal load bearing mode of the lumbar spine (1), which has been widely used to treat diseases such as disc degeneration, degenerative lumbar instability, vertebral spondylolisthesis, extensive decompression of spinal stenosis, and lumbar space infection (2).

Minimally invasive spinal (MIS) techniques, such as minimally invasive transforaminal lumbar interbody fusion 
Page 2 of 13

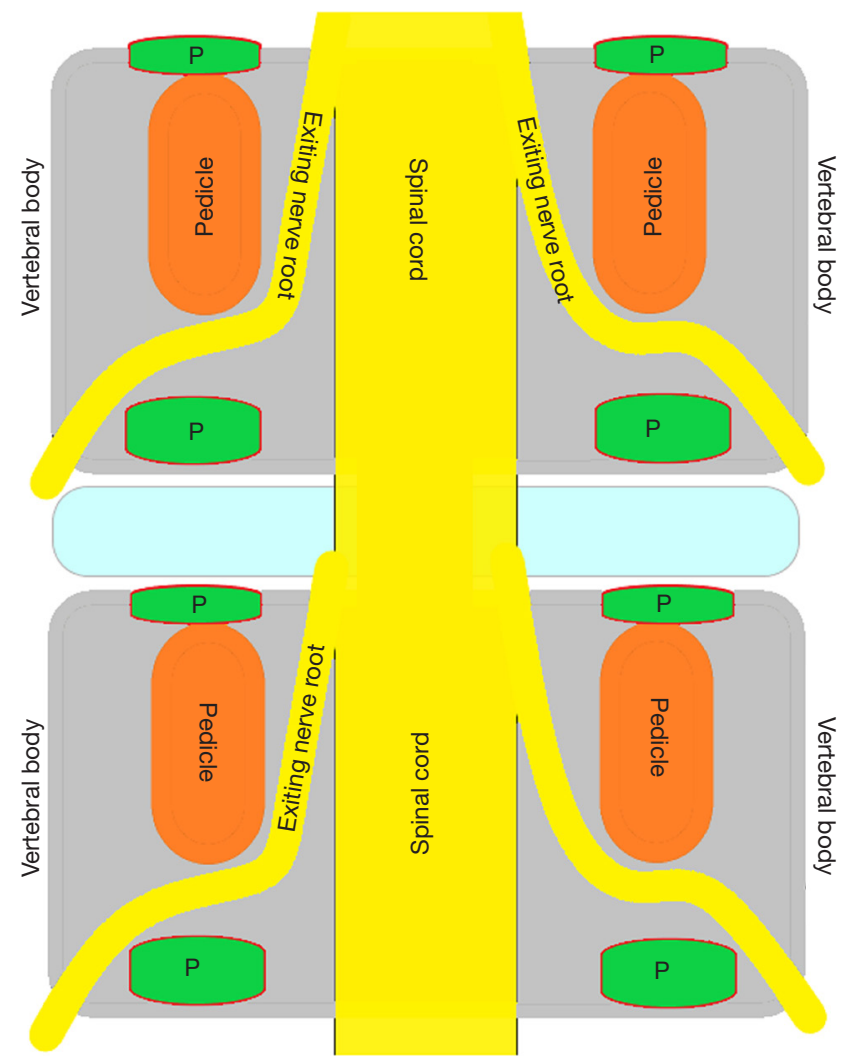

Figure 1 Illustration of the screw entry point of oblique fixation from posterior corner in lumbar spine.

(MIS-TLIF), extreme lateral lumbar interbody fusion (XLIF), oblique lateral interbody fusion (OLIF), axial lumbar interbody fusion (AxiaLIF), offer comparable results with the traditional open lumbar interbody fusion with the benefits of a shorter hospital stay, less blood loss, and shorter recovery time.

Currently, endoscopic transforaminal decompression and interbody fusion (ETDIF) has been widely discussed due to its advantages of less trauma, less bleeding, quick recovery, high safety, and relatively fewer complications, as well as adverse factors such as incomplete decompression, steep learning curve, low fusion rate, and high radiation risk (3). Furthermore, this technique requires the use of supplemental posterior pedicle-screw. Decompression, interbody fusion and percutaneous pedicle screw implantation are not completed in a single $7.5-\mathrm{mm}$ channel. So far, there has been no correlational study, which complete nerve decompression, interbody fusion and interbody fixation in one stop under spinal endoscopy technology. Percutaneous endoscopic transforaminal
Chen et al. Oblique fixation from posterior corner in lumbar spine.

oblique fixation from posterior corner in lumbar spine (PETOFPC) overcomes the above limitations, and realizes one-stop decompression, fusion and fixation in a single regular minimally invasive channel.

The purpose of this paper is to confirm the anatomical feasibility for PETOFPC in the posterolateral transforaminal approach and to provide anatomic data for the design of new integrated fixable and fused interbody cage. We present the following article in accordance with the STROBE reporting checklist (available at http://dx.doi. org/10.21037/atm-20-2046).

\section{Methods}

\section{Study design and materials}

As a cohort study, sixty normal adult volunteers (22 men and 38 women), who do not have lumbar tumors, trauma, deformity or a history of lumbar surgery, underwent CT scan (slice thickness $1.0 \mathrm{~mm}$ ) of the lumbar spine with GE Light Speed 64CT in our hospital from June 1, 2019 to Dec 31, 2019. The volunteers' age was from 20 to 51 years, with an average age of 33.8 years. The male volunteers' height was 168 to $182 \mathrm{~cm}$, with an average of $174.6 \mathrm{~cm}$, and the female volunteers' height was 157 to $171 \mathrm{~cm}$, with an average of $165.3 \mathrm{~cm}$. All scan information was transmitted to the GE AW4.4 workstation for measurement.

The study was approved by The Institutional Ethics Committees of Shandong Provincial Hospital Affiliated to Shandong University (No. 2019-252) and informed consent was taken from all the patients.

\section{Measurement parameters}

To investigate fully radiographic anatomy of PETOFPC, the authors chose to measure the distances from the posterior corner (P point, Figure 1) in lumbar spine to the corresponding targets.

Posterior corner in lumbar spine was selected as the screw entry point $(\mathrm{P}$ point) for oblique fixation. The screw entry point $(\mathrm{P}$ point) in posterior-inferior corner is the bony area between the upper margin of the pedicle and the upper endplate. The screw entry point (P point) in posteriorsuperior corner is the bony area between the lower margin of the pedicle and the lower endplate (Figure 2). The corresponding targets are A, B, C, D, E, and F, respectively (A: the middle point of the contralateral anterior-middle $1 / 3, \mathrm{~B}$ : the contralateral anterior-middle point, $\mathrm{C}$ : the middle point 


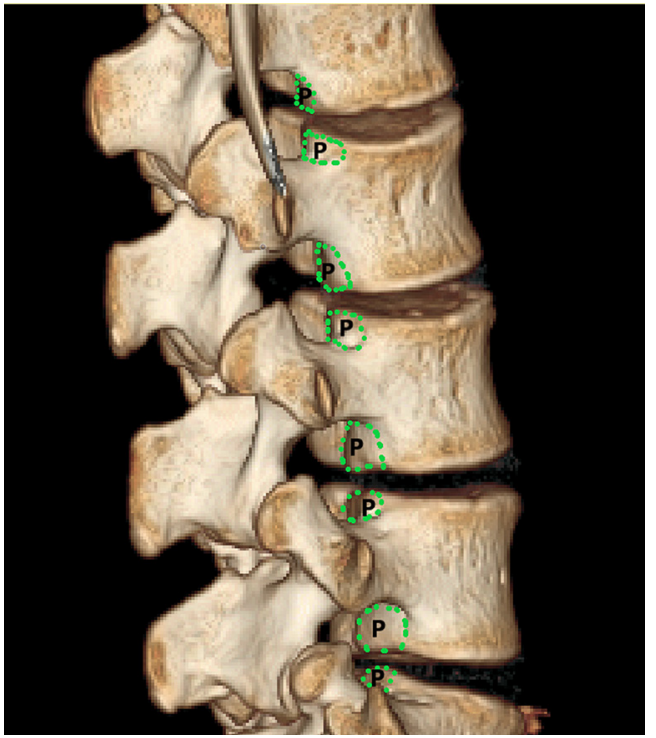

Figure 2 The screw entry point in three-dimensional CT reconstructing in the GE AW4.4 workstation.

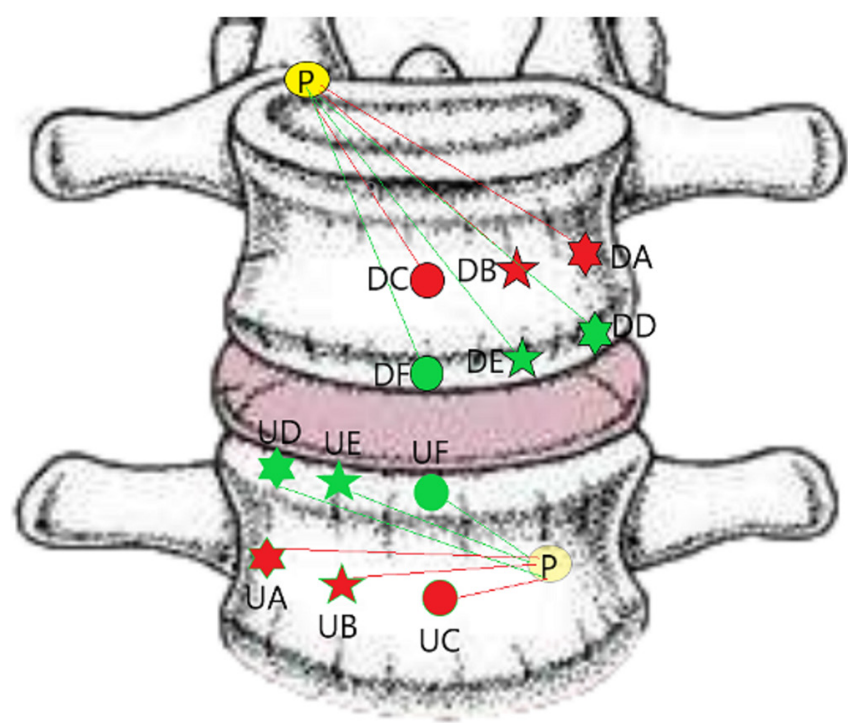

Figure 3 Illustration of six paths of oblique fixation from posterior corner in lumbar spine.

of the contralateral side, D: the upper point of contralateral anterior-middle $1 / 3, \mathrm{E}$ : the contralateral anterior-upper point, $\mathrm{F}$ : the middle-upper point of the contralateral side) in the contralateral anterior region (Figure 3).

In the process of oblique fixation from posterior corner in lumbar spine, each path could be divided into two groups, medium group and full-length group. The medium group

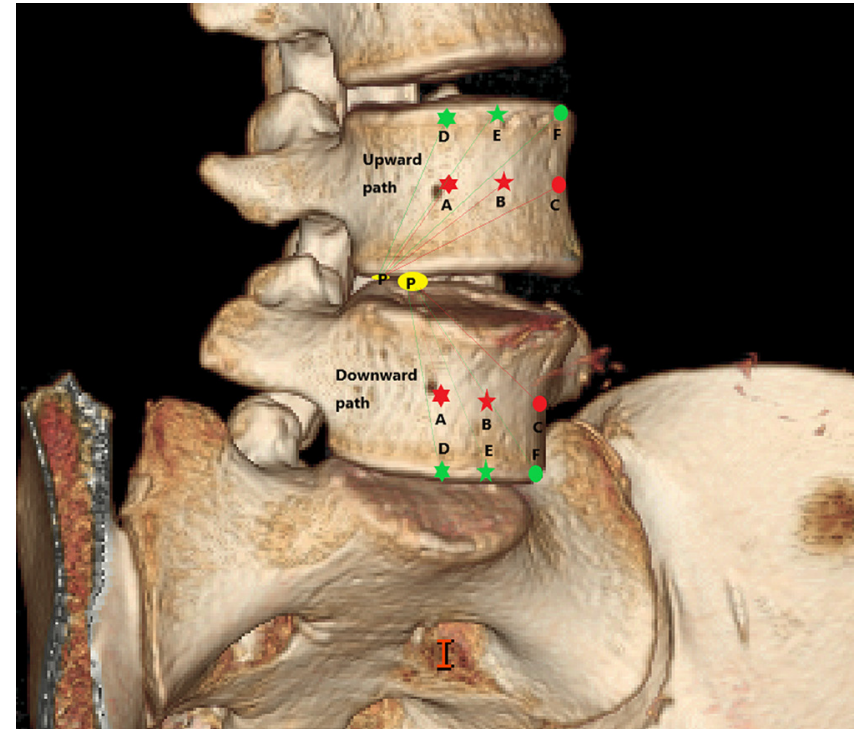

Figure 4 Six paths in three-dimensional CT reconstructing in the GE AW4.4 workstation.

included PA, PB and PC, while the full-length group included PD, PE and PF (Figure 4). The upward paths included PUA, PUB, PUC, PUD, PUE, and PUF. The downward paths included PDA, PDB, PDC, PDD, PDE, and PDF.

PUA path: distance from the posterior-lower corner of the upper vertebral body to the middle point of the contralateral anterior-middle $1 / 3$.

PUB path: distance from the posterior-lower corner of the upper vertebral body to the contralateral anteriormiddle point.

PUC path: distance from the posterior-inferior corner of the upper vertebral body to the middle point of the contralateral side.

PUD path: distance from the posterior-lower corner of the upper vertebral body to the upper point of contralateral anterior-middle $1 / 3$.

PUE path: distance from the posterior-lower corner of the upper vertebral body to the contralateral anterior-upper point.

PUF path: distance from the posterior-lower corner of the upper vertebral body to the middle-upper point of the contralateral side.

PDA path: distance from the posterior-upper corner of the lower vertebral body to the middle point of the contralateral anterior-middle $1 / 3$.

PDB path: distance from the posterior-upper corner of the lower vertebral body to the contralateral anteriormiddle point. 
Page 4 of 13

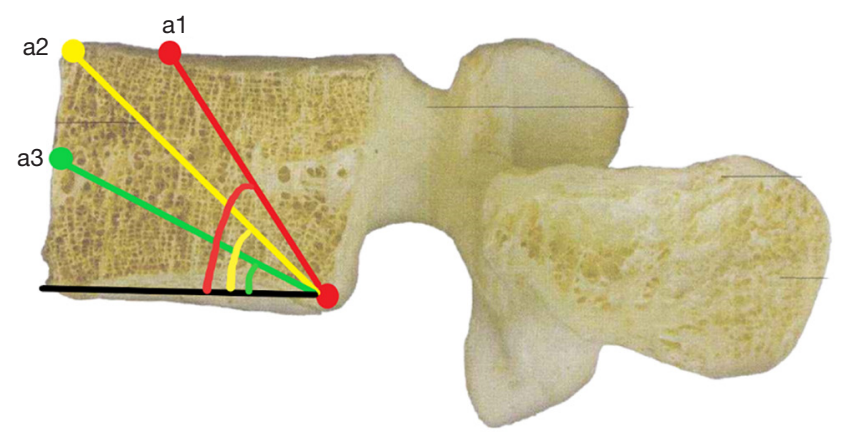

Figure 5 Illustration of the angles between the upward paths and the parallel line of the corresponding vertebral endplate in sagittal plane.

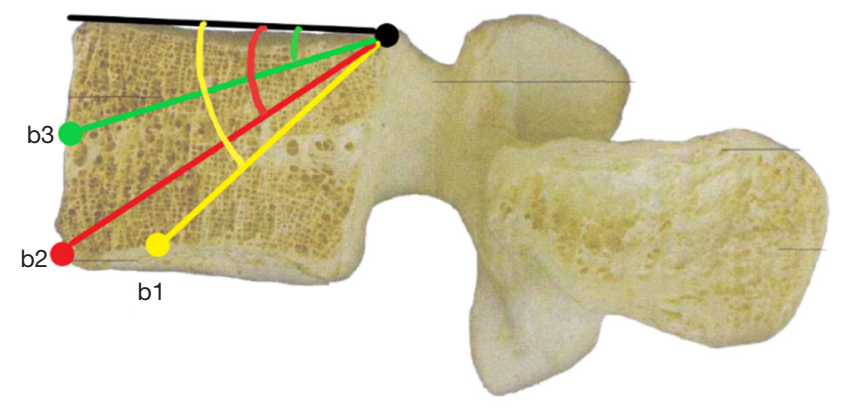

Figure 6 Illustration of the angles between the downward paths and the parallel line of the corresponding vertebral endplate in sagittal plane.

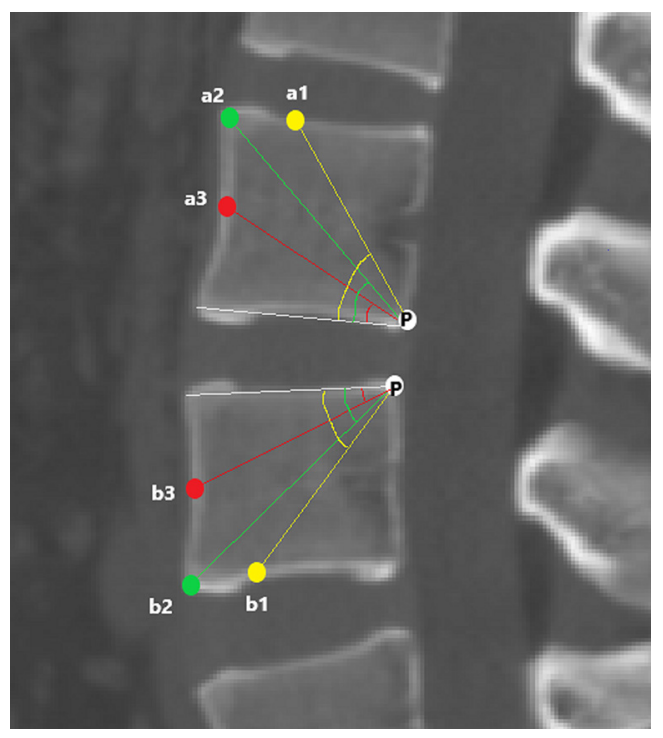

Figure 7 The angles measured in sagittal plane in the GE AW4.4 workstation.
Chen et al. Oblique fixation from posterior corner in lumbar spine.

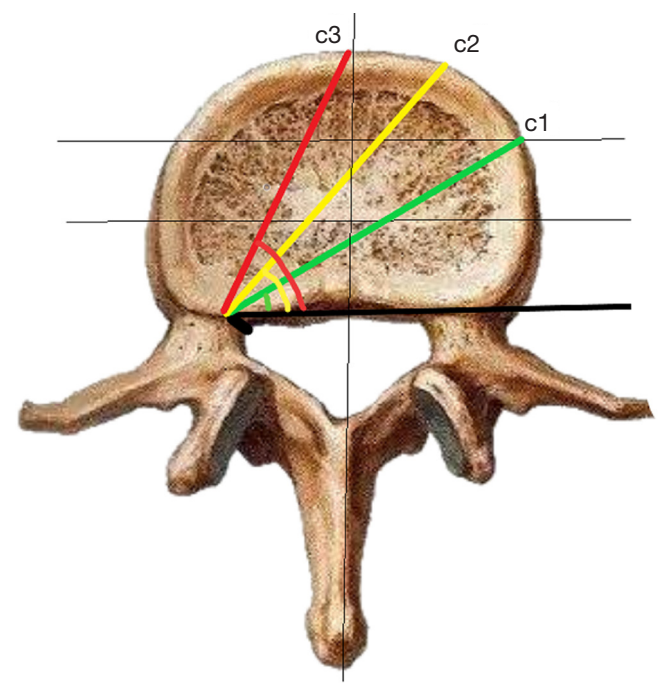

Figure 8 Illustration of the angles between the paths and the tangent line to the posterior border of the vertebral body in axial plane.

PDC path: distance from the posterior-upper corner of the lower vertebral body to the middle point of the contralateral side.

PDD path: distance from the posterior-upper corner of lower vertebral body to the upper point of the contralateral anterior-middle $1 / 3$.

PDE path: distance from the posterior-upper corner of lower vertebral body to the contralateral anterior-lower point.

PDF path: distance from posterior-upper corner of lower vertebral body to the middle-lower point of the contralateral side.

The angles of oblique fixation from posterior corner in lumbar spine in sagittal and axial planes (Figures 5-9).

a1: the angle between the line, which is from the posteriorlower corner of the upper vertebral body to front-middle $1 / 3$ of the upper endplate, and lower endplate parallel line.

a2: the angle between the line, which is from the posterior-lower corner of the upper vertebral body to anterior-upper corner, and lower endplate parallel line.

a3: the angle between the line, which is from the posteriorlower corner of the upper vertebral body to the midpoint of the anterior border, and lower endplate parallel line.

b1: the angle between the line, which is from the posterior-upper corner of the lower vertebral body to front-middle $1 / 3$ of the lower endplate, and upper endplate parallel line.

b2: the angle between the line, which is from the posterior-upper corner of the lower vertebral body to 
anterior-lower corner, and upper endplate parallel line.

b3: the angle between the line, which is from the posteriorupper corner of the lower vertebral body to the midpoint of the anterior border, and upper endplate parallel line.

c1: the angle between the projection, which is generated from the posterior corner of the upper vertebral body to contralateral front-middle $1 / 3$ on the endplate, and the tangent line to the posterior border of the vertebral body.

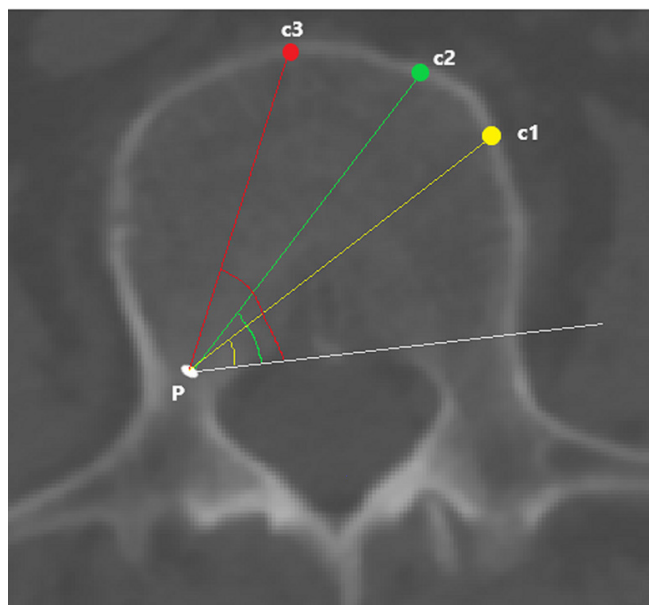

Figure 9 The angles measured in axial plane in the GE AW4.4 workstation. c2: the angle between the projection, which is generated from the posterior corner of the upper vertebral body to contralateral anterior corner on the endplate, and the tangent line to the posterior border of the vertebral body.

c3: the angle between the projection, which is generated from the posterior corner of the upper vertebral body to the midpoint of the anterior border on the endplate, and the tangent line to the posterior border of the vertebral body.

Two orthopedic surgeons (worked for 9 years and 11 years) and one radiographic physician (worked for 15 years) used a double-blind method to measure the above data. Each doctor took measurements once, and the average of the three measurements was used as the final data.

\section{Statistical methods}

The measured data, including 3,600 length data and 2,880 angle data, were counted and compared with the independent sample t test by SPSS25.0 statistical software.

\section{Results}

\section{The lengths of each path (Table 1)}

In the medium group, PC path was the shortest $(38.56 \pm 3.25 \mathrm{~mm}), \mathrm{PA}$ path and $\mathrm{PB}$ path had little difference

Table 1 Each path length for oblique fixation from the posterior corner in lumbar spine

\begin{tabular}{|c|c|c|c|c|c|c|c|}
\hline Path (mm) & L1-U & L2-U & L3-U & L4-U & L5-U & $\mathrm{F}$ value & $P$ value \\
\hline \multirow[t]{2}{*}{$\mathrm{PA}^{\dagger}$} & $37.87 \pm 3.48^{*}$ & $40.04 \pm 2.75$ & $40.96 \pm 2.98$ & $41.36 \pm 3.20$ & $42.11 \pm 4.15$ & 9.759 & $\leq 0.001$ \\
\hline & $39.51 \pm 3.32$ & $39.96 \pm 2.75$ & $40.76 \pm 2.99$ & $41.27 \pm 3.20$ & $42.47 \pm 4.22$ & & \\
\hline \multirow[t]{2}{*}{$\mathrm{PB}^{\dagger}$} & $38.95 \pm 3.29$ & $40.86 \pm 3.24$ & $41.61 \pm 3.01$ & $41.28 \pm 3.50$ & $41.06 \pm 3.81$ & 2.963 & 0.002 \\
\hline & $40.71 \pm 3.39$ & $40.78 \pm 3.24$ & $41.42 \pm 3.02$ & $41.19 \pm 3.47$ & $41.42 \pm 3.88$ & & \\
\hline $\mathrm{PC}^{\dagger}$ & $38.95 \pm 3.23$ & $39.08 \pm 2.82$ & $38.90 \pm 2.75$ & $38.43 \pm 3.47$ & $38.30 \pm 3.76$ & & \\
\hline \multirow[t]{2}{*}{$P D^{\ddagger}$} & $43.20 \pm 3.09$ & $45.57 \pm 2.73$ & $46.19 \pm 2.82$ & $46.03 \pm 2.97$ & $46.50 \pm 3.83$ & 8.220 & $\leq 0.001$ \\
\hline & $45.12 \pm 3.12$ & $45.31 \pm 2.64$ & $45.50 \pm 2.82$ & $45.73 \pm 2.98$ & $47.80 \pm 4.10$ & & \\
\hline $\mathrm{PE}^{\ddagger}$ & $44.15 \pm 2.96$ & $46.30 \pm 3.09$ & $46.76 \pm 2.88$ & $45.96 \pm 3.27$ & $45.54 \pm 3.55$ & 3.371 & $\leq 0.001$ \\
\hline $\mathrm{PF}^{\ddagger}$ & $44.63 \pm 3.03$ & $44.53 \pm 2.62$ & $43.83 \pm 2.61$ & $43.19 \pm 3.17$ & $44.13 \pm 3.60$ & & \\
\hline
\end{tabular}

${ }^{*}$, the length in the table consists of mean and standard deviation; ${ }^{\dagger}, \mathrm{PA}, \mathrm{PB}$ and $\mathrm{PC}$ belong to the medium group; ${ }^{\ddagger}, \mathrm{PD}, \mathrm{PE}$ and $\mathrm{PF}$ belong to the full-length group. L, lumbar; $S$, sacrum; $U$, the "upward" path; $D$, the "downward" path. PA/PB/PC/PD/PD/PF, from P point to targets $\mathrm{A} / \mathrm{B} / \mathrm{C} / \mathrm{D} / \mathrm{E} / \mathrm{F}$. 


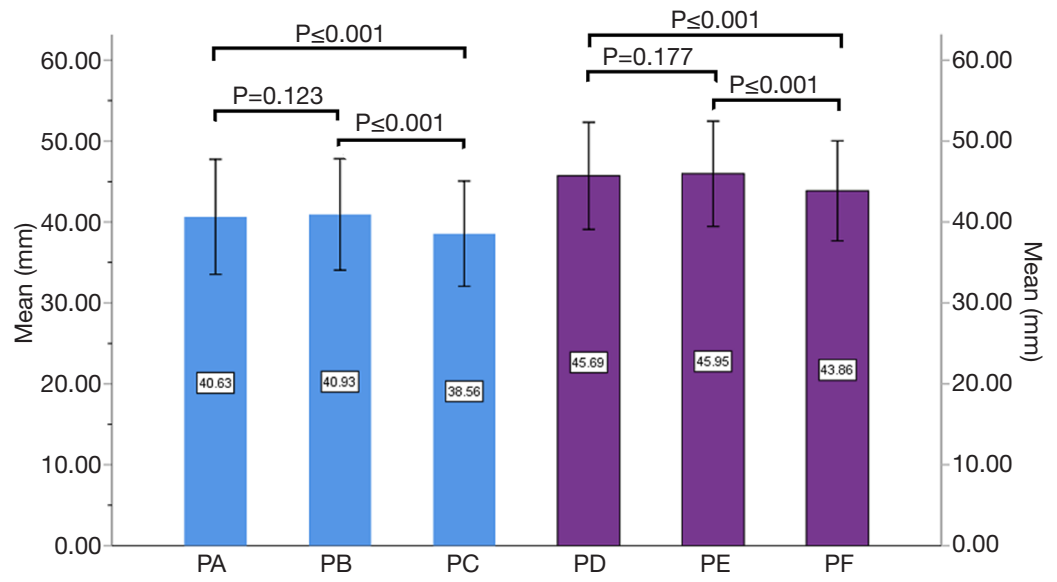

Figure 10 The length comparison of each path for oblique fixation from posterior corner in lumbar spine.
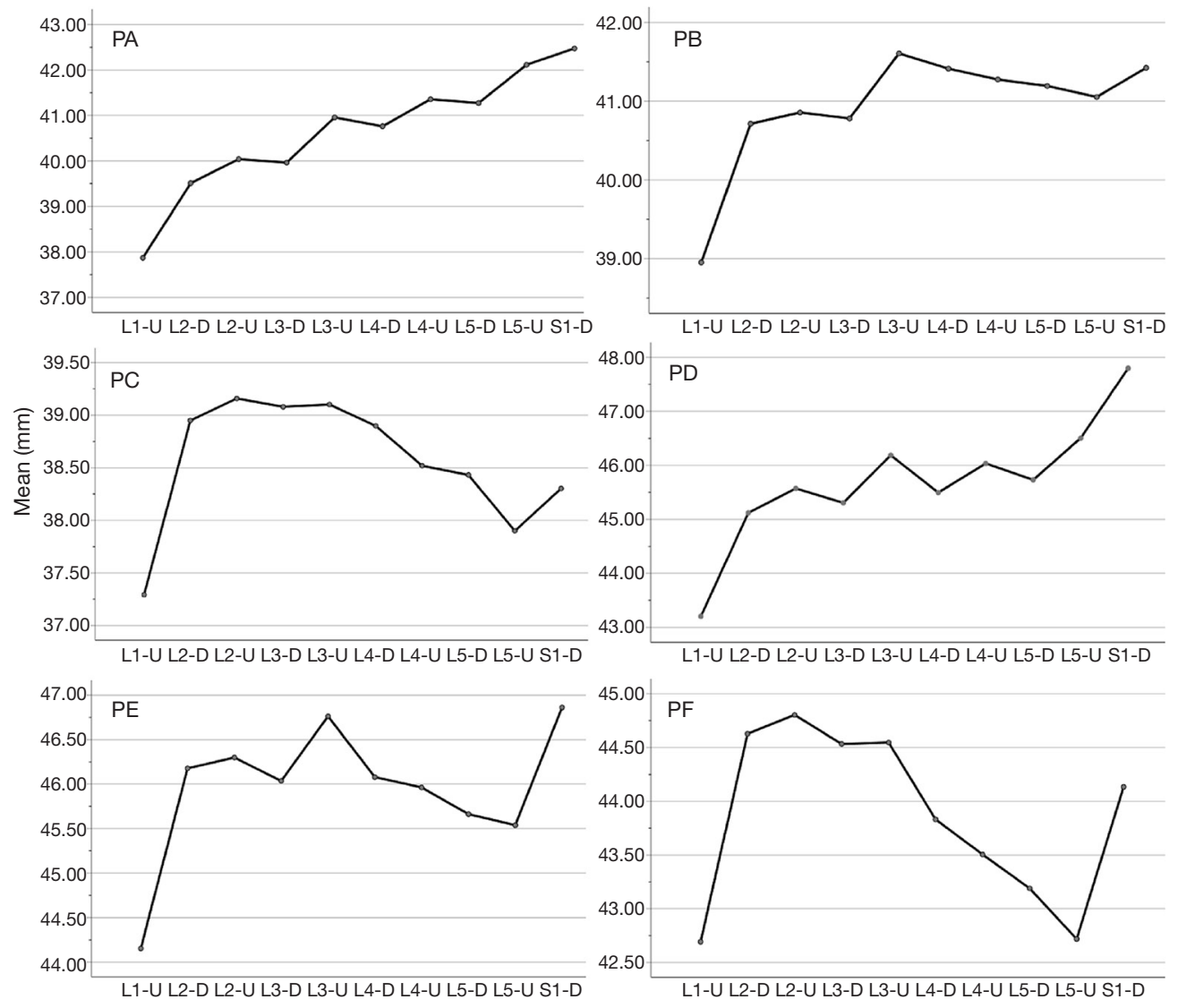

Figure 11 Variation trend of the length of each path from L1 to S1.

$(\mathrm{P}=0.123)$, and there was no statistical significance. In the full-length group, PF path was the shortest $(43.86 \pm 3.09 \mathrm{~mm})$, and there was no significant difference between $\mathrm{PD}$ path and $\mathrm{PE}$ path $(\mathrm{P}=0.177)$ (Figure 10). All the paths increased successively from L1 to S1, among which $\mathrm{PA}$ and PD paths were the most obvious, followed by $\mathrm{PB}$ and PE paths. PC path and PF path first increased and then decreased (Figures 11,12). 


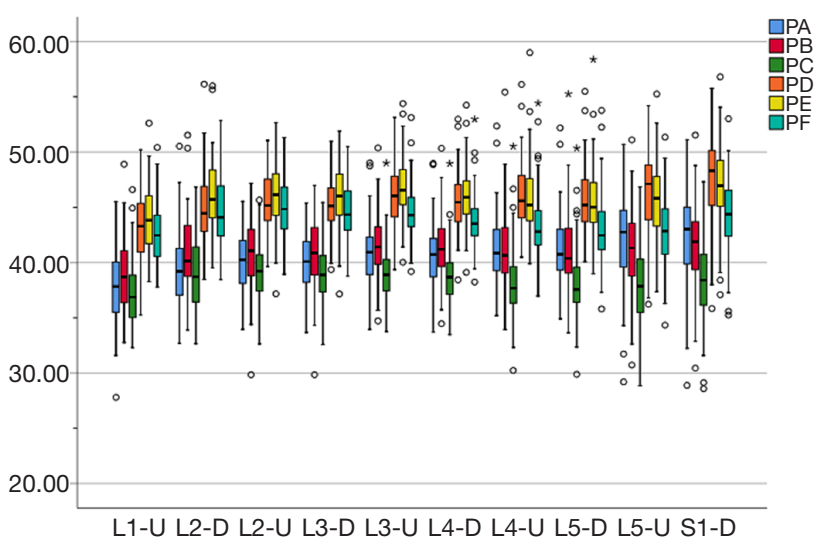

Figure 12 The length variation of the upward and downward paths from L1 to S1. * denotes the extreme value, which has no statistical significance.

\section{The angles of each path (Table 2)}

The included angles a1, a2, a3, b1, b2, b3 and c1, c2 and $\mathrm{c} 3$ in the sagittal plane were significantly different $(\mathrm{P} \leq 0.001)$ (Figure 13) $(\mathrm{a} 1>\mathrm{a} 2>\mathrm{a} 3, \mathrm{~b} 1>\mathrm{b} 2>\mathrm{b} 3, \mathrm{c} 1<\mathrm{c} 2$ $<\mathrm{c} 3)$. In the angles of sagittal down-path, b1, b2 and b3 change significantly and consistently, showing a trend of decreasing first and then increasing, which is the smallest at L3 $\left(46.08 \pm 4.37^{\circ}, 36.21 \pm 3.51^{\circ}, 22.47 \pm 3.01^{\circ}\right)$ and the largest at $\mathrm{S} 1\left(69.49 \pm 8.28^{\circ}, 61.23 \pm 7.74^{\circ}, 36.60 \pm 5.00^{\circ}\right)$. In the angles of sagittal up-path, a1 and a 2 change in the same way, showing an increasing trend. While a 3 goes down and then goes up, which is the smallest at L4 $\left(23.43 \pm 4.29^{\circ}\right)$ and the largest at L5 $\left(24.36 \pm 2.94^{\circ}\right)$. In the angles of axial plane, $\mathrm{c} 1, \mathrm{c} 2$ and $\mathrm{c} 3$ change in the same way, showing a trend

Table 2 Each path angle for oblique fixation from posterior corner in lumbar spine ( $\left.{ }^{\circ}\right)$

\begin{tabular}{|c|c|c|c|c|c|c|c|c|c|}
\hline Plane & Angle & L1 & L2 & L3 & L4 & L5 & S1 & $F$ value & $P$ value \\
\hline \multirow[t]{6}{*}{ Sagittal $^{\dagger}$} & a1 & $50.19 \pm 4.50$ & $50.43 \pm 4.09$ & $51.15 \pm 4.18$ & $51.17 \pm 3.97$ & $54.21 \pm 4.33$ & & 8.770 & $\leq 0.001$ \\
\hline & a2 & $39.21 \pm 4.39$ & $39.37 \pm 3.72$ & $40.00 \pm 4.26$ & $39.72 \pm 4.06$ & $42.03 \pm 4.58$ & & 4.392 & 0.002 \\
\hline & a3 & $24.70 \pm 3.20$ & $24.26 \pm 2.96$ & $23.81 \pm 3.06$ & $23.43 \pm 4.29$ & $24.36 \pm 2.94$ & & 1.337 & 0.256 \\
\hline & b1 & & $46.79 \pm 3.73$ & $46.08 \pm 4.37$ & $47.13 \pm 3.99$ & $48.33 \pm 3.61$ & $69.49 \pm 8.28$ & 237.613 & $\leq 0.001$ \\
\hline & b2 & & $36.39 \pm 3.39$ & $36.21 \pm 3.51$ & $36.95 \pm 3.47$ & $39.30 \pm 3.76$ & $61.23 \pm 7.74$ & 268.915 & $\leq 0.001$ \\
\hline & b3 & & $23.01 \pm 2.34$ & $22.47 \pm 3.01$ & $22.88 \pm 2.40$ & $23.94 \pm 2.61$ & $36.60 \pm 5.00$ & 212.251 & $\leq 0.001$ \\
\hline \multirow[t]{3}{*}{ Axial $^{\ddagger}$} & c1 & $39.17 \pm 2.76$ & $40.07 \pm 2.75$ & $39.28 \pm 2.65$ & $37.9 \pm 2.44$ & $36.01 \pm 2.90$ & $33.87 \pm 2.94$ & 44.275 & $\leq 0.001$ \\
\hline & c2 & $54.08 \pm 3.66$ & $55.1 \pm 3.32$ & $54.22 \pm 3.25$ & $52.92 \pm 3.48$ & $50.25 \pm 3.27$ & $47.92 \pm 3.54$ & 39.329 & $\leq 0.001$ \\
\hline & c3 & $71.39 \pm 5.89$ & $72.35 \pm 4.24$ & $73.11 \pm 2.10$ & $72.49 \pm 2.64$ & $70.5 \pm 2.54$ & $67.15 \pm 3.60$ & 20.298 & $\leq 0.001$ \\
\hline
\end{tabular}

${ }^{*}, \mathrm{P}<0.05$, the comparison between groups is statistically significant; ${ }^{\dagger}, \mathrm{a} 1, \mathrm{a} 2, \mathrm{a} 3, \mathrm{~b} 1, \mathrm{~b} 2$ and b3 are measured in sagittal plane; ${ }^{\ddagger}, \mathrm{c} 1, \mathrm{c} 2$ and c3 are measured in axial plane. L, lumbar; S, sacrum.

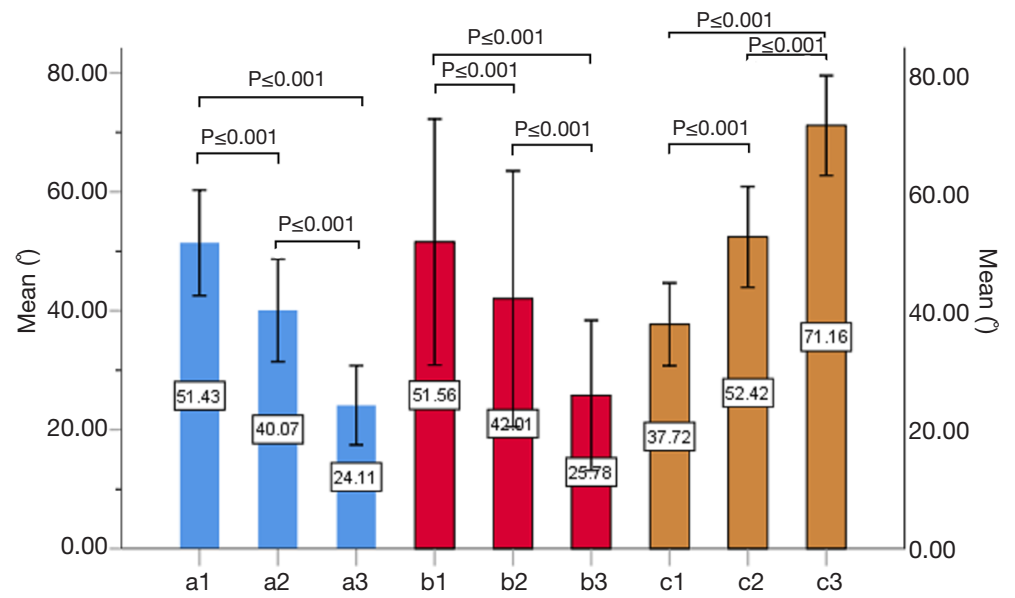

Figure 13 The angle comparison of each path for oblique fixation from posterior corner in lumbar spine. 

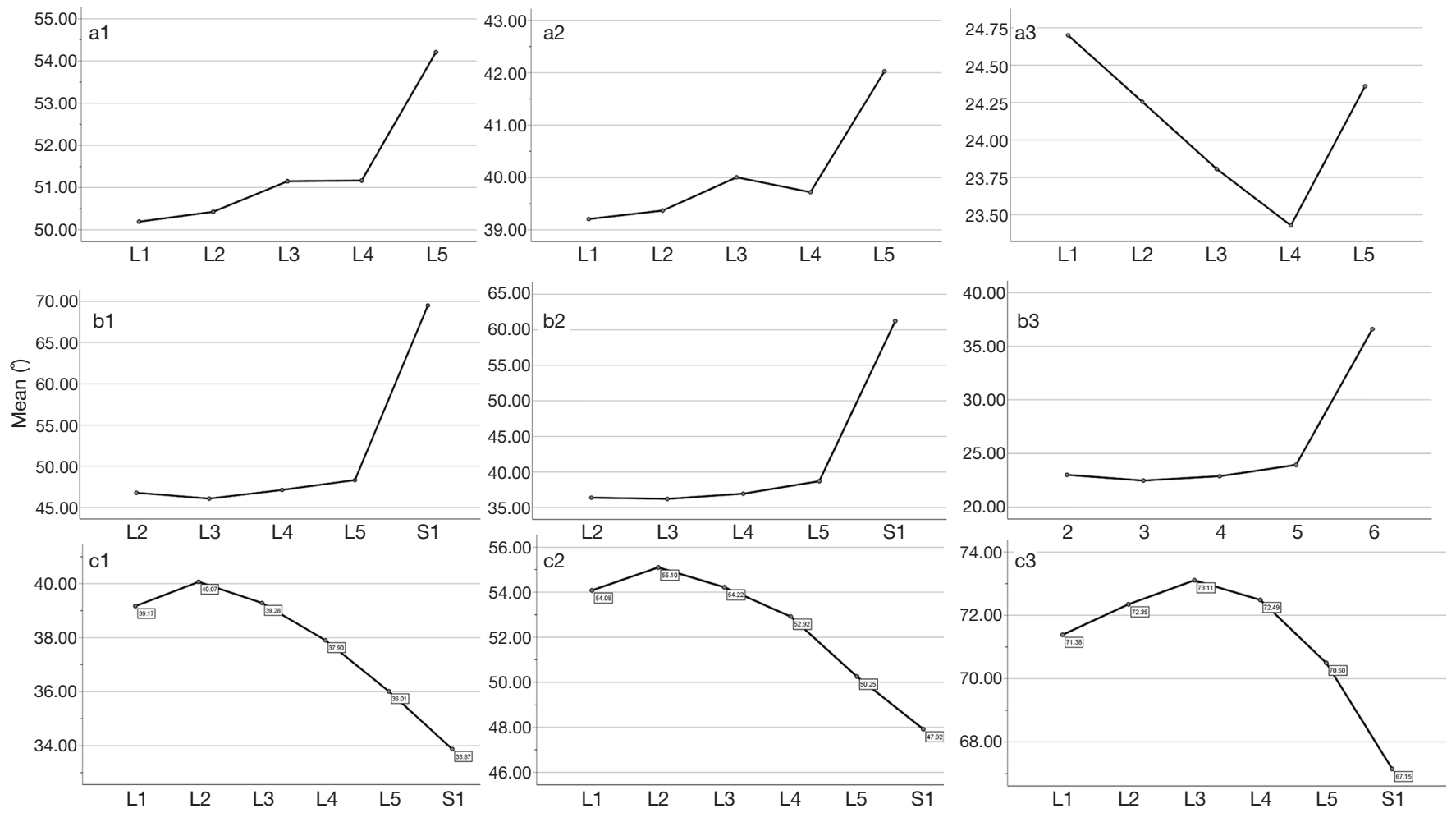

Figure 14 Variation trend of each angle from L1 to S1.

of increasing first and then decreasing. $\mathrm{c} 1$ and $\mathrm{c} 2$ are the largest at $\mathrm{L} 2\left(40.07 \pm 2.75^{\circ}, 55.1 \pm 3.32^{\circ}\right)$, and $\mathrm{c} 3$ is the largest at L3 $\left(73.11 \pm 2.10^{\circ}\right) . \mathrm{c} 1, \mathrm{c} 2$ and $\mathrm{c} 3$ are the smallest at $\mathrm{S} 1$ $\left(33.87 \pm 2.94^{\circ}, 47.92 \pm 3.54^{\circ}, 67.15 \pm 3.60^{\circ}\right)$ (Figures 14,15$)$.

\section{Discussion}

Several studies (4-7) have shown that MIS techniques, such as MIS-TLIF, XLIF, OLIF, AxiaLIF, offer comparable results with the traditional open lumbar interbody fusion with the benefits of a shorter hospital stay, less blood loss, and shorter recovery time. But all these procedures have different attributes in terms of disruption of the normal anatomic structures; collateral damage to the structures in the neighborhood; accessibility to the different levels of the spine (8).

ETDIF was first reported in 2012 by Osman (8). This article confirms the least invasive (arthroscopic) lumbar decompression, interbody fusion (with bone morphogenetic protein-2 and allograft bone chips), and percutaneous pedicle screw instrumentation consistently produced satisfactory results in all demographics. Several studies (9-15) have proved the feasibility of this technique and achieved satisfactory clinical outcomes. Lee et al. (13) reported 18 patients were operated on with the described technique without any posterior fixation between 2001 and 2007, but interbody fusion occurred ideally in 10 patients and the non-interbody fusion rate is as high as $44.4 \%$. As early as in 2013, some scholars (15) also found the high complication rate of $36 \%$ among 57 patients who were treated with was due to the use of non-expandable device and lack of posterior fixation, thus it can be seen percutaneous transforaminal endoscopic lumbar interbody fusion (PT-Endo-LIF) technique requires the use of supplemental posterior pedicle-screw based instrumentation for added stability.

In ETDIF/PT-Endo-LIF technique, percutaneous pedicle screw implantation uses different approach from interbody decompression and fusion. Moreover, decompression and fusion are often accomplished by setting a 14-mm channel additionally. Decompression, interbody fusion and percutaneous pedicle screw implantation are not completed in a single $7.5-\mathrm{mm}$ channel. PETOFPC overcomes the above limitations, and realizes one-stop decompression, fusion and fixation in a single $7.5-\mathrm{mm}$ channel (Figure 16). The implementation of this technique 

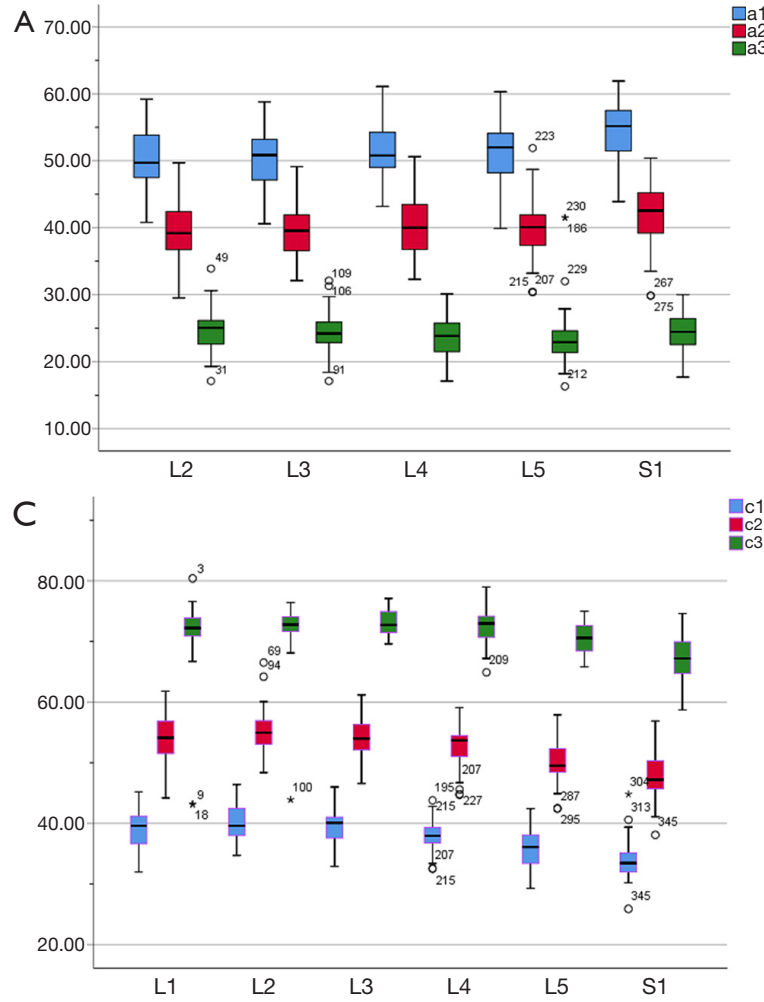

Figure 15 The angle variation in sagittal and axial planes from L1 to S1. * denotes the extreme value, which has no statistical significance.

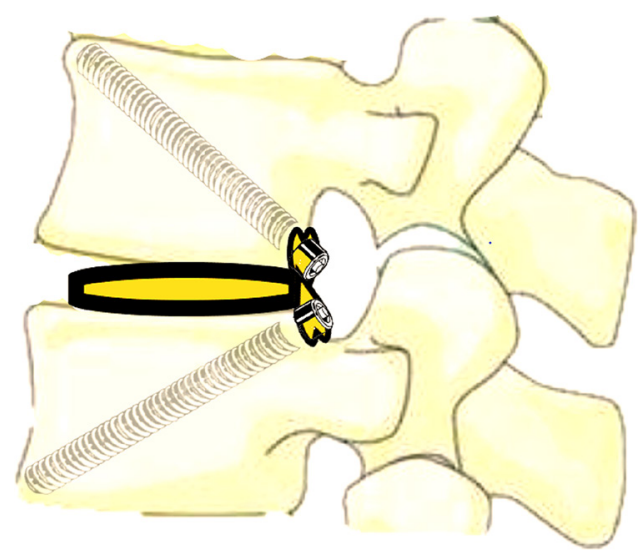

Figure 16 Illustration of one-stop decompression, fusion, and fixation.

requires the participation of a new integrated fixable and fused interbody cage (Figure 17).

PETOFPC technique innovatively sets screws from posterior corner in lumbar spine in a regular $7.5-\mathrm{mm}$ diameter channel. PETOFPC is also performed in the posterolateral transforaminal approach (Figure 18). A

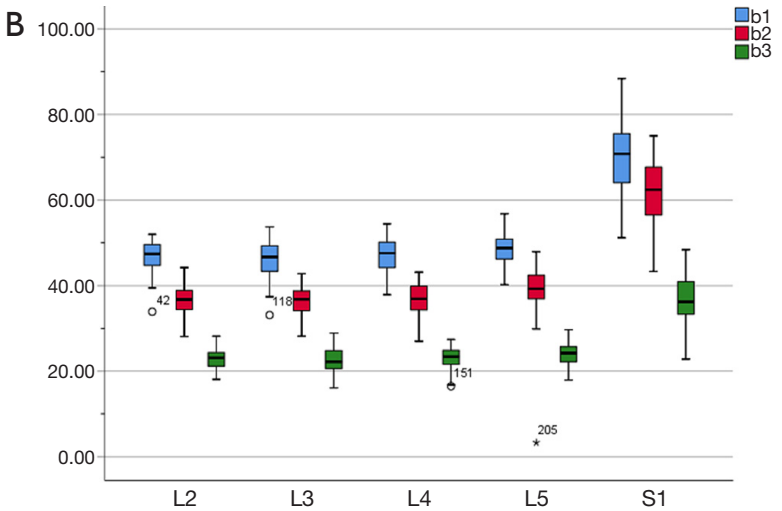

cylindrical sheath with an outer diameter of $7.5 \mathrm{~mm}$ were inserted in sequence. Through the sheath alone or the endoscope, the intervertebral disk and cartilaginous endplate were removed while confirming through percutaneous endoscopic observation that no cartilage remained. At the completion of the endplate preparation, the new integrated fixable and fused interbody cage was inserted into the intervertebral space (16). Before the new integrated fixable and fused interbody cages were expanded, we should clearly expose the screw entry point in posterior corner. The screw entry point is the bony area between the pedicle and the endplate. When intervertebral height was restored, the two wings of new integrated fixable and fused interbody cage splay to the sides and fit to posterior corner in lumbar spine (Figure 19). In the single 7.5 -mm channel, endoscopic visualization was used to monitor placing screws to make the new integrated fixable and fused interbody cage fixed in lumbar spine. The angles of screw placement were made up of inward inclined angle, head tilt angle and tail tilt angle, namely c1, c2, c3, a1, a2, a3, b1, b2 and b3, all which was measured and analyzed in this study.

In this article, we preset six paths as screw trajectory of oblique fixation from posterior corner in lumbar spine, 


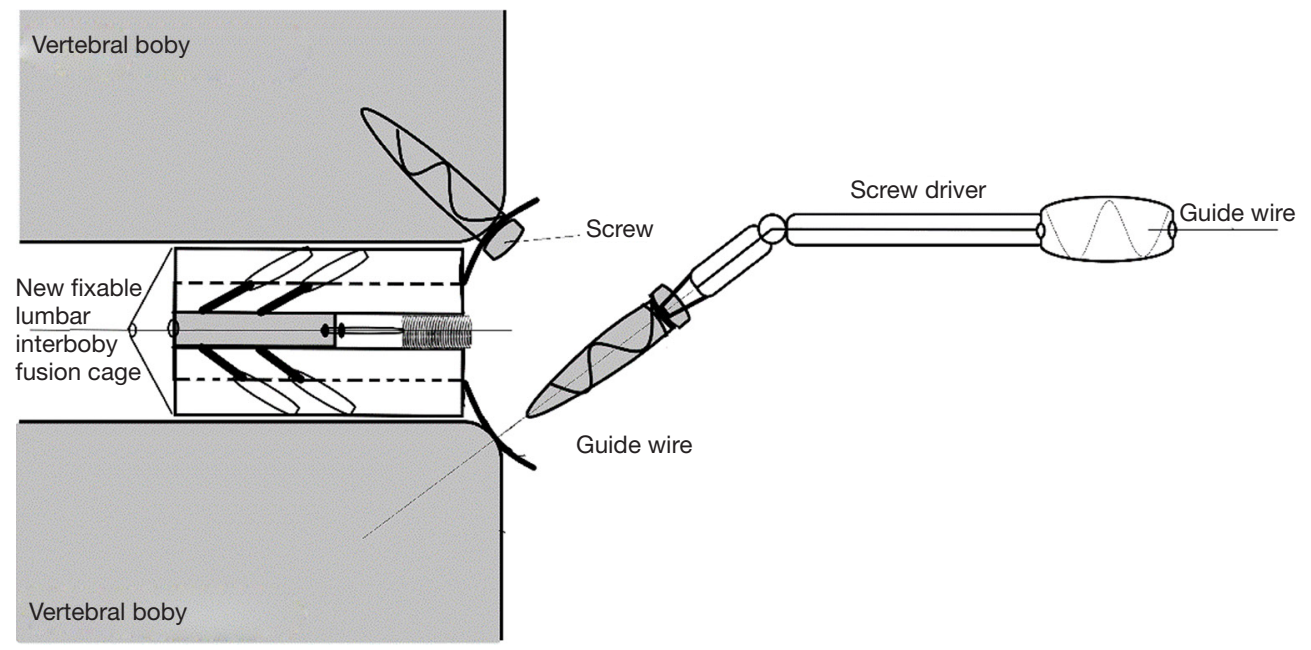

Figure $\mathbf{1 7}$ The schematic diagram of new expandable fixable interbody cage.

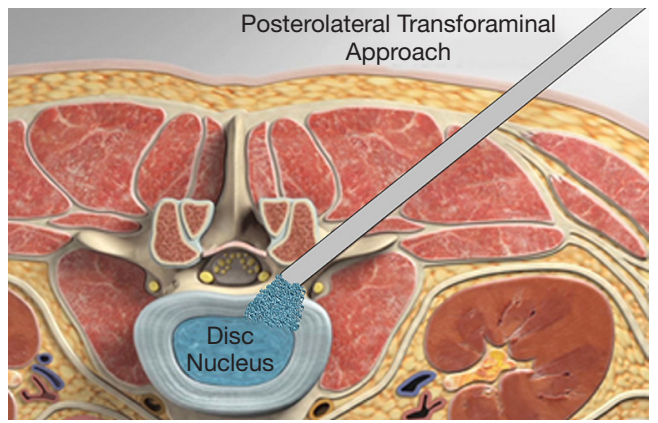

Figure 18 The posterolateral transforaminal approach performed in PETOFPC technology. PETOFPC, percutaneous endoscopic transforaminal oblique fixation from posterior corner in lumbar spine.

namely, PA, PB, PC, PD, PE, and PF (Figures 3,4). The design of the pathway is determined by the technical characteristics of spinal endoscopy in clinical practice, considering simultaneously the biomechanical properties of the screw, the longer bone canal and more cortical penetration. The paths should be neither penetrating into the intervertebral space nor making the anterior large vessels injured. Considering the above factors, PE path should be the optimal (longest) path for oblique fixation from posterior corner in lumbar spine.

$\mathrm{PE}$ path was the longest in all the paths for oblique fixation from posterior corner in lumbar spine, with an average length of $45.95 \mathrm{~mm}$, which was significantly longer than other paths in the same vertebral body (Figures 10-12). Its upward path first increases and then decreases, reaching the highest point at $\mathrm{L} 3(46.76 \pm 2.88 \mathrm{~mm})$. Its downward path first decreases and then increases, with the lowest decline at L5 $(45.66 \pm 3.21 \mathrm{~mm})$ (Figure 20). At the same time, the fixed angles (a2, b2, c2) of the PE path in sagittal and axial planes are between a1-a3, b1-b3, and c1-c3, which may neither penetrate into the intervertebral space nor injure the anterior large vessels. Therefore, the PE path is safe and easy to grasp in theory.

In addition, the pullout strength of PE path is greater because the bone canal was across the entire vertebral body from the posterior (upper and lower) corner of one side in lumbar spine oblique to the anterior (lower and upper) corner of the contralateral side in lumbar spine. The PE path is considered to be the optimal one for oblique fixation from posterior corner in lumbar spine.

Youn et al. (14) investigated that PT-Endo-LIF is suitable for no more than II level lumbar spondylolisthesis. Lee et al. (13) also stated that ETDIF is not suitable for patients with severe central and lateral recess stenosis as endoscopic ventral decompression of soft disc can only achieve limited decompression. Osteoporosis needs to be carefully managed with further pedicle screws fixation to prevent subsidence and fracture of endplate.

Although conventional fusion surgery is a gold standard for lumbar interbody fusion, we have performed PETOFPC only in limited patients who have spinal instability that should be corrected but are not able to undergo general anesthesia or prefer only for "local anesthetic" procedure, especially in elderly patients. Generally speaking, PETOFPC seems to be a promising surgical technique for treating patients 

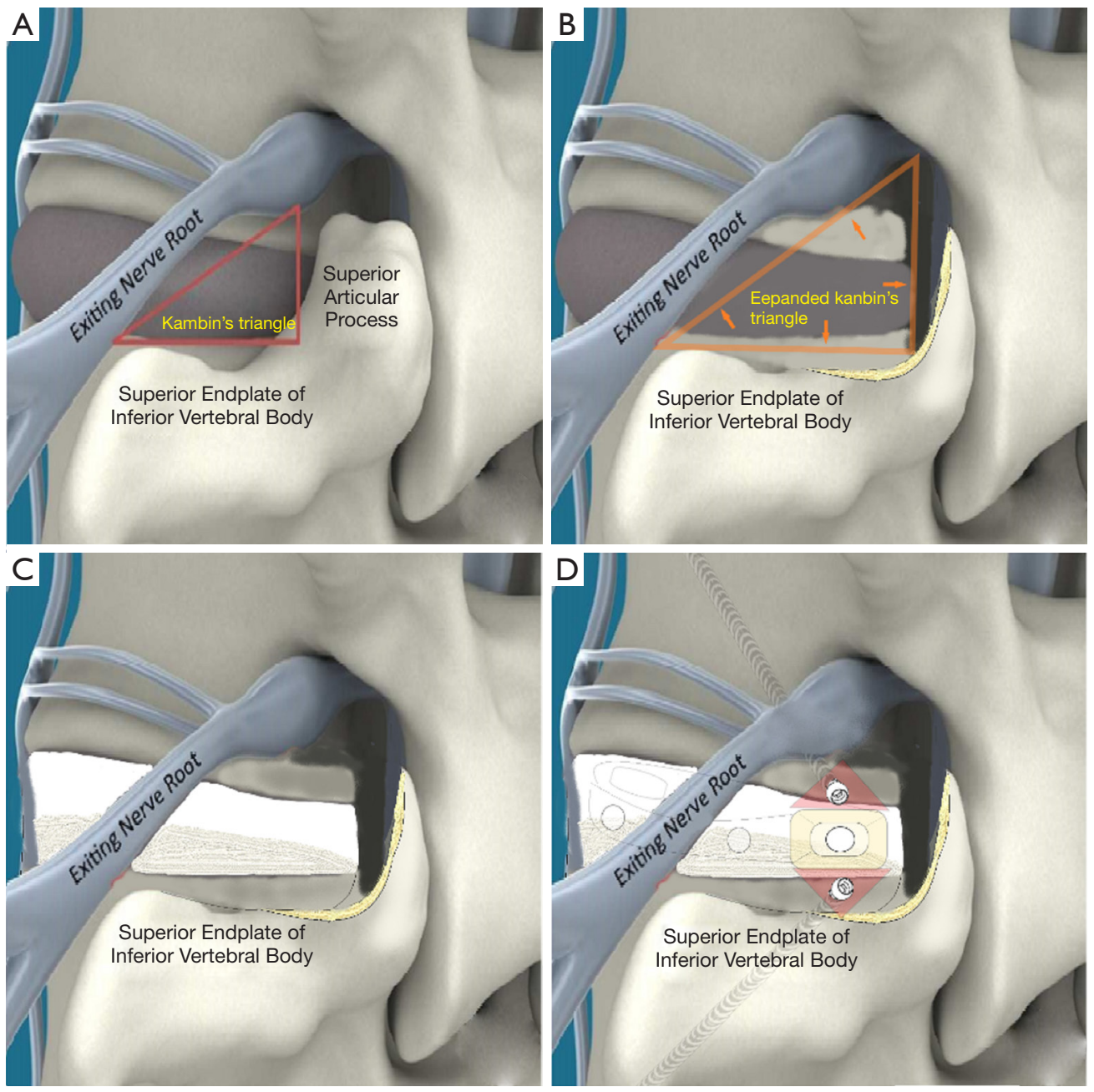

Figure 19 Flow diagram of percutaneous endoscopic transforaminal oblique fixation from posterior corner in lumbar spine. (A) Kambin's triangle before Foraminoplasty. (B) Kambin's triangle after Foraminoplasty. (C) Complete decompression through Kambin's triangle. (D) One-stop decompression, fusion, and fixation with new integrated fixable and fused interbody cage.

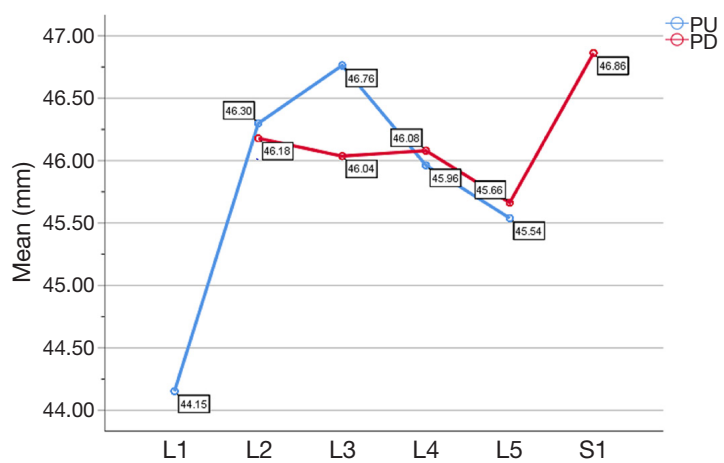

Figure 20 Variation trend of the PE path. suffering from lumbar disc herniation (LDH), lumbar spinal stenosis (LSS) and mild lumbar spondylolisthesis (LS). It was necessary that postoperative patients were treated with braces in order to facilitate lumbar interbody fusion.

Our study has some limitations that should be discussed. First, the radiographic measurement was not compared with the dry bone measurement, and the correlation analysis between each index and body length is not carried out, which will be improved in the subsequent study. In addition, the biomechanical evaluation of compressive stress, stretch stress, torsion stress and fatigue load between the lumbar spine should be made in order to obtain objective evaluation for its bearing capacity before the new integrated fixable and fused interbody cage is produced. Finally, three-dimensional 


\section{Page 12 of 13}

finite element analysis and biomechanical evaluations are also required after the cage model is completed.

\section{Conclusions}

In this study, the lengths and angles of each path for posterior corner in lumbar spine are measured comprehensively, which confirms anatomic feasibility for PETOFPC and provides anatomic data for the design of new integrated fixable and fused interbody cage. PETOFPC may be a very promising technology and have great clinical significance when further biomechanical evaluations, favorable clinical outcomes in larger groups of patients and long-term follow-up examinations are completed.

\section{Acknowledgments}

Funding: Supported by The Key Research and Development Program of Shandong Province: 2018GSF118074.

\section{Footnote}

Reporting Checklist: The authors have completed the STROBE reporting checklist. Available at http://dx.doi. org/10.21037/atm-20-2046

Data Sharing Statement: Available at http://dx.doi. org/10.21037/atm-20-2046

Peer Review File: Available at http://dx.doi.org/10.21037/ atm-20-2046

Conflicts of Interest: All authors have completed the ICMJE uniform disclosure form (available at http://dx.doi. org/10.21037/atm-20-2046). The authors have no conflicts of interest to declare.

Ethical Statement: The authors are accountable for all aspects of the work in ensuring that questions related to the accuracy or integrity of any part of the work are appropriately investigated and resolved. The study was approved by The Institutional Ethics Committees of Shandong Provincial Hospital Affiliated to Shandong University (No. 2019-252) and informed consent was taken from all the patients.

Open Access Statement: This is an Open Access article distributed in accordance with the Creative Commons
Chen et al. Oblique fixation from posterior corner in lumbar spine.

Attribution-NonCommercial-NoDerivs 4.0 International License (CC BY-NC-ND 4.0), which permits the noncommercial replication and distribution of the article with the strict proviso that no changes or edits are made and the original work is properly cited (including links to both the formal publication through the relevant DOI and the license). See: https://creativecommons.org/licenses/by-nc-nd/4.0/.

\section{References}

1. Arai $\mathrm{Y}$, Takahashi M, Kurosawa H, et al. Comparative study of iliac bone graft and carbon cage with local bone graft in posterior lumbar interbody fusion. J Orthop Surg (Hong Kong) 2002;10:1-7.

2. Mai GH, Chang ZL, Duan W, et al. Analysis of early results of transforaminal lumbar interbody fusion. China Journal of Endoscopy 2008;14:231-3.

3. Ahn Y, Youn MS, Heo DH. Endoscopic transforaminal lumbar interbody fusion: a comprehensive review. Expert Rev Med Devices 2019;16:373-80.

4. Pearson AM, Lurie JD, Tosteson TD, et al. Who should undergo surgery for degenerative spondylolisthesis? Treatment effect predictors in SPORT. Spine (Phila Pa 1976) 2013;38:1799-811.

5. Shunwu F, Xing Z, Fengdong Z, et al. Minimally invasive transforaminal lumbar interbody fusion for the treatment of degenerative lumbar diseases. Spine (Phila Pa 1976) 2010;35:1615-20.

6. Kim JS, Jung B, Lee SH. Instrumented Minimally Invasive Spinal-Transforaminal Lumbar Interbody Fusion (MISTLIF): Minimum 5-Year Follow-Up with Clinical and Radiologic Outcomes. Clin Spine Surg 2018;31:E302-9.

7. Kim TY, Kang KT, Yoon DH, et al. Effects of lumbar arthrodesis on adjacent segments: differences between surgical techniques. Spine (Phila Pa 1976) 2012;37:1456-62.

8. Osman SG. Endoscopic transforaminal decompression, interbody fusion, and percutaneous pedicle screw implantation of the lumbar spine: A case series report. Int J Spine Surg 2012;6:157-66.

9. Lewandrowski KU, Ransom NA, Ramírez León JF, et al. The Concept for A Standalone Lordotic Endoscopic Wedge Lumbar Interbody Fusion: The LEW-LIF. Neurospine 2019;16:82-95.

10. Ahn Y, Youn MS, Heo DH. Endoscopic transforaminal lumbar interbody fusion: a comprehensive review. Expert Rev Med Devices 2019;16:373-80.

11. Yang JC, Hai Y, Ding Y, et al. Percutaneous endoscopic transforaminal lumbar interbody fusion for lumbar spinal 
stenosis. Zhonghua Yi Xue Za Zhi 2018;98:3711-5.

12. Wang MY, Grossman J. Endoscopic minimally invasive transforaminal interbody fusion without general anesthesia: initial clinical experience with 1-year followup. Neurosurg Focus 2016;40:E13.

13. Lee SH, Erken HY, Bae J. Percutaneous Transforaminal Endoscopic Lumbar Interbody Fusion: Clinical and Radiological Results of Mean 46-Month Follow-Up. Biomed Res Int 2017;2017:3731983.

Cite this article as: Chen F, Liu X, Sun J, Xin J, Su C, Wang G, Cui X. Radiographic anatomy and clinical significance of percutaneous endoscopic transforaminal oblique fixation from posterior corner in lumbar spine. Ann Transl Med 2020;8(21):1391. doi: 10.21037/atm-20-2046
14. Youn MS, Shin JK, Goh TS, et al. Full endoscopic lumbar interbody fusion (FELIF): technical note. Eur Spine J 2018;27:1949-1955.

15. Jacquot F, Gastambide D. Percutaneous endoscopic transforaminal lumbar interbody fusion: is it worth it? Int Orthop 2013;37:1507-10.

16. Nakamura S, Taguchi M. Full Percutaneous Lumbar Interbody Fusion: Technical Note. J Neurol Surg A Cent Eur Neurosurg 2017;78:601-6. 